\title{
Active Gathering of Frictional Properties from Objects
}

\author{
Carlos Rosales $^{1}$, Arash Ajoudani ${ }^{2}$, Marco Gabiccini ${ }^{3}$ and Antonio Bicchi ${ }^{4}$
}

\begin{abstract}
This work proposes a representation that comprises both shape and friction, as well as the exploration strategy to gather them from an object. The representation is developed under a common probabilistic framework, particularly it uses a Gaussian Process to approximate the distribution of the friction coefficient over the surface, also represented as a Gaussian Process. The surface model is exploited to compute straight lines (geodesic flows) that guide the exploration. The exploration follows these flows by employing an impedance controller in pursuance of safety, shape accommodation and contact enforcement, while measuring the necessary data to estimate the friction coefficient. The exploratory probes consist of an RGBD camera and an Intrinsic Tactile sensor (ITs) mounted on a robotic arm. Experimental results give evidence for the effectiveness of the algorithm in the friction coefficient gathering and enrichment of the object representation.
\end{abstract}

\section{INTRODUCTION}

Objects are perceived by robotic systems using noncontact or contact sensing, or a combination of both, for tasks such as recognition, and grasping and manipulation. Object properties which are of interested in robotics include shape, color, weight, texture, friction coefficients, inertial values, degrees of freedom, among many others. Shape and color are predominant due to the extensive use of cameras and the large computer vision field. However, other properties, such as the friction coefficients, are relevant to object grasping and manipulation and object recognition, by itself or in combination with visual information [1].

Visual and tactile array information has been successfully integrated into a single object shape model for different purposes. For instance, [2] uses the tactile information to discriminate object pose hypothesis coming from vision, [3] defines a hierarchical strategy to explore the object using the tactile information to refine the object shape only when necessary. In more recent works, [4] fuses the two sources of information by applying symmetry constraints to reconstruct the complete object shape, and [5] uses the contact points to improve the object pose within the hand, initially obtained

1 C. Rosales is with Centro di Ricerca "E. Piaggio", Università di Pisa, 1 Largo L. Lazzarino, 56122 Pisa, Italy. \{carlos.rosales\} at for.unipi.it

2 A. Ajoudani is with the Department of Advanced Robotics (ADVR), Istituto Italiano di Tecnologia, Genova, Italy \{arash.ajoudani\} at iit.it

${ }^{3}$ M. Gabiccini is with the Centro di Ricerca "E. Piaggio", Università di Pisa, 1 Largo L. Lazzarino, 56122 Pisa, Department of Civil and Industrial Engineering (DICI), Largo Lucio Lazzarino 1, Pisa and Department of Advanced Robotics (ADVR), Italian Institute of Technology, 30 Via Morego, 16163 Genova, Italy. m.gabiccini at ing.unipi.it

${ }^{4} \mathrm{~A}$. Bicchi is with the Centro di Ricerca "E. Piaggio", Università di Pisa, 1 Largo L. Lazzarino, 56122 Pisa and Department of Advanced Robotics (ADVR), Italian Institute of Technology, 30 Via Morego, 16163 Genova, Italy. bicchi at centropiaggio.unipi.it

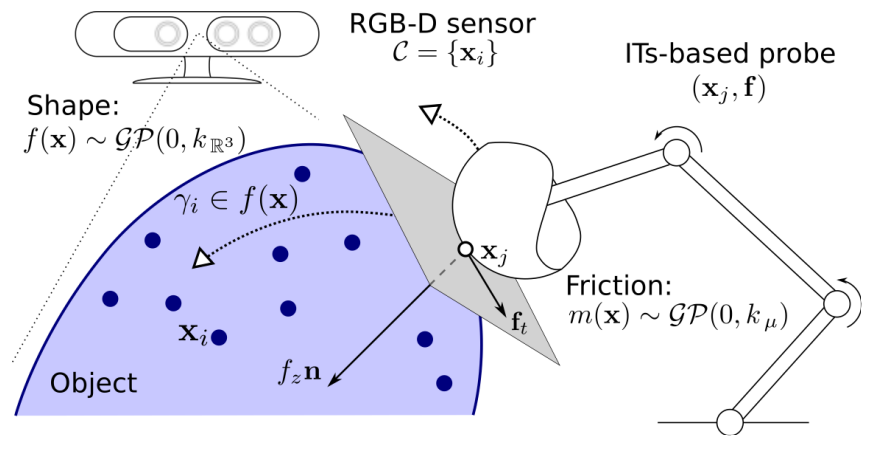

Fig. 1: The shape is acquired by an RGBD sensor and the friction coefficients by the intrinsic tactile sensor, both shape $f(\mathbf{x})$ and friction $m(\mathbf{x})$ functions are represented as a Gaussian process $\mathcal{G P}(0, k(\cdot))$. The exploration follows geodesic flows $\gamma_{i}$ on the surface of a fixed object using a compliant behavior.

by vision. Nonetheless, other object properties apart from the shape are hardly explored, perhaps due to the fact that one needs specific sensory systems to measure them and a proper representation to process the information. In this preliminary work, the hardware setup resembles that of [6] (see Fig. 1), but instead of using tactile arrays at the tip of the exploratory probe, we use an ITs because of its capability to retrieve the contact force [7], which is necessary to our approach.

This work proposes a methodology to gather the kinetic coefficient of friction, a potential feature for object recognition [8], and certainly relevant to grasp determination [9], as well as its representation over the surface. The representation is developed under a probabilistic framework, particularly it uses Gaussian processes (GPs), which has proved to provide good results in object recognition [10] and grasp planning [11], [12]. The shape model allows to compute paths on the surface, here we use straight lines, hence the name geodesic flows, to guide the exploration. The actual exploration is executed by the ITs mounted on a $7 \mathrm{DoF}$ arm. To this end, a controlled compliant behavior should provide a safe contact, shape accommodation and good data quality acquisition, assuming that the object is fixed to the table. The rest of the paper is organized as follows: Section II describes the devices and type of information they provide, Section III details the object representation and the exploration strategy, Section IV shows the experimental results, and, finally, Section V draws the conclusions and points for future developments. 


\section{SENSORY DATA}

As shown in Fig. 1, the setup is composed of an RGBD sensor and an ITs mounted on a robot's end-effector, and a fixed object to be explored. Visual and tactile data acquired from them are described below.

\section{A. Visual data}

The object surface is acquired using a commercial RGBD sensor (Fig. 2a). The raw data comes in a point cloud format and it is pre-processed to isolate the point cloud of the object, $\mathcal{C}=\left\{\mathbf{x}_{1}, \ldots, \mathbf{x}_{N}\right\}$, where $\mathbf{x}_{i} \in \mathbb{R}^{3}$ is a point on the surface. It is also possible to estimate the normal at the contact points which will be useful in the object representation learning (Subsection III-A). The extrinsic camera calibration is performed using objects as patterns and performing 3D pose estimation.

\section{B. Tactile data}

The ITs is composed of a force/torque (F/T) sensor, a tip and a compliant structure as a safety mechanism due to the high cost of the F/T sensor (Fig. 2b). The tactile data includes the contact point on the object surface, $\mathbf{x}_{j} \in \mathbb{R}^{3}$ w.r.t. the F/T sensor frame, and the contact force $\mathbf{f}_{j} \in \mathbb{R}^{3}$ expressed in the contact frame, such that we are able to estimate the kinetic coefficient of friction as

$$
\mu=\frac{\left\|\mathbf{f}_{t}\right\|}{\left\|f_{z}\right\|},
$$

where $f_{z}$ and $\mathbf{f}_{t}$ are the components in the normal and in the tangent plane at the contact point, respectively [13]. There is no general correlation between the static and kinetic coefficients of friction [14], however, in general, tables are available for pairs of materials [13]. Given a set of materials to be encountered in the environment and the fingertip material, and assuming a fixed condition (humidity, temperature, etc.), one might query for the kinetic coefficient of friction and select the best matching pair and obtain the static coefficient of friction. In this work, we will present results with the the former only, since the procedure is equally applicable for both of them. The tip is $3 \mathrm{D}$ printed in ABS and with a hemi-spherical shape. The latter simplifies the contact point and force calculation, but other ellipsoidal surfaces can be used as well [7], or even arbitray NURBS surfaces that require iterative optimization routines.

The ITs is mounted on a 7 DoF KUKA robotic arm to form the exploratory probe with high mobility. The compliant mechanism is an in-parallel passive compliant coupler (PPCC) with known Cartesian stiffness, and used to estimate the deformation using the $\mathrm{F} / \mathrm{T}$ measurements. However, the stiffness is set with spring preloads to have negligible deformations on the range of forces applied during the exploration.

\section{EXPLORATION STRATEGY}

The proposed strategy is based on three main ingredients: the object representation for a complemented sensory data processing, exploration paths for an optimal information

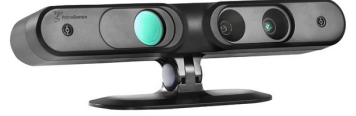

(a) RGBD sensor.

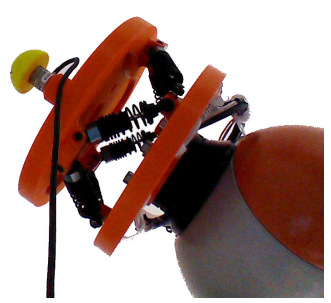

(b) Intrinsic Tactile sensor.
Fig. 2: Visual and tactile devices.

gain, and contour following for a successful data acquisition. Details on each of them are presented next.

\section{A. Object Representation: Gaussian Process for implicit functions}

The object shape is modeled by an implicit function, $f(\mathbf{x})=0$, with $\mathbf{x} \in \mathbb{R}^{3}$ being any point on the surface. This function is approximated using a Gaussian process (GP) [15] over the object point cloud, $\mathcal{C}$, as

$$
f(\mathbf{x}) \sim \sum_{i=1}^{N} \alpha_{i} k_{\mathbb{R}_{3}}\left(\mathbf{x}, \mathbf{x}_{i}, \sigma\right),
$$

where $k_{\mathbb{R}_{3}}(\cdot)$ is the covariance function centered at points $\mathbf{x}_{i} \in \mathcal{C}, \sigma$ is a smoothing parameter (set according to the units of $\mathbf{x}$ and enforced to have the same value for all terms), and $\alpha_{i}$ is the weighing factor for each term (to be learned from the measurements).

For 3D points, we use a triangular position kernel (see [10], [16] for more details), which has the form

$$
k_{\mathbb{R}_{3}}(\mathbf{d}, \sigma)= \begin{cases}1-\frac{\left(\mathbf{d}^{\top} \mathbf{d}\right)^{\frac{1}{2}}}{2 \sigma} & \text { if }\left(\mathbf{d}^{\top} \mathbf{d}\right)^{\frac{1}{2}} \leq 2 \sigma \\ 0 & \text { if }\left(\mathbf{d}^{\top} \mathbf{d}\right)^{\frac{1}{2}}>2 \sigma,\end{cases}
$$

where we perform the change of variable $\mathbf{d}=\mathbf{x}-\mathbf{x}_{i}, \forall i$.

The friction coefficient value over the surface is also modeled by an implicit function, $m(\mathbf{d})$, and approximated as well by a GP, as

$$
m(\mathbf{d}) \sim \sum_{i=1}^{N} \beta_{i} k_{\mu}(\mathbf{d}, \Sigma) .
$$

Due to the nature of the data, we decide to use a squared exponential as the covariance function instead, with the form

$$
k_{\mu}(\mathbf{d}, \Sigma)=e^{-\frac{\left(\mathbf{d}^{\top} \mathbf{d}\right)}{2 \Sigma}}
$$

where $\Sigma$ is a smoothing factor without units. It is worth noting that, the points on the surface in this case come from both the tactile and visual data (Section II).

The determination of the weights $\alpha_{i}$ and $\beta_{i}$ depends on the function we want to represent to choose proper labels to the sensory data. In the case of the object surface, we would like to model

$$
f(\mathbf{x}) \begin{cases}>0, & \text { if } \mathbf{x} \text { is outside the surface } \\ =0, & \text { if } \mathbf{x} \text { is on the surface } \\ <0, & \text { if } \mathbf{x} \text { is inside the surface }\end{cases}
$$




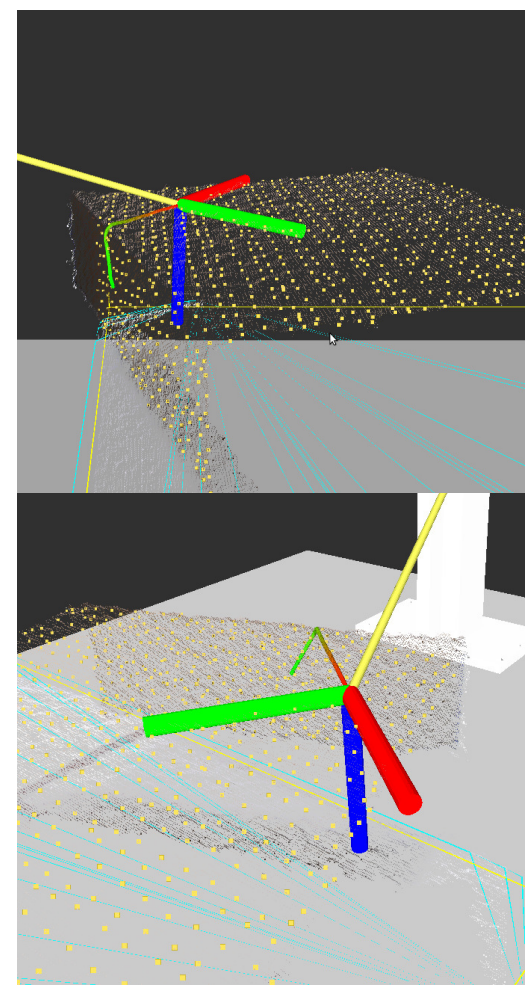

(a) The box has flat surfaces, hence geodesics are straight lines.

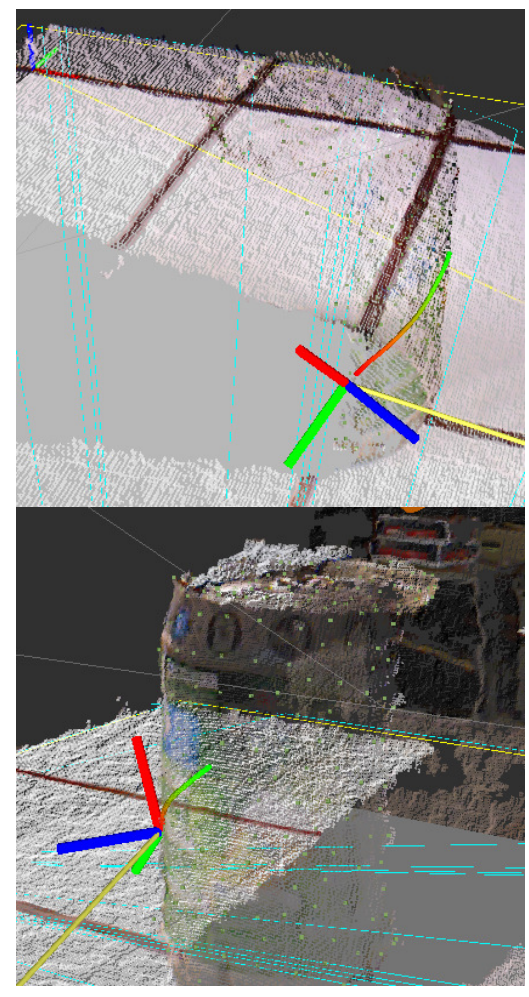

(b) The can is almost a cylinder, hence geodesics are elliptical curves.

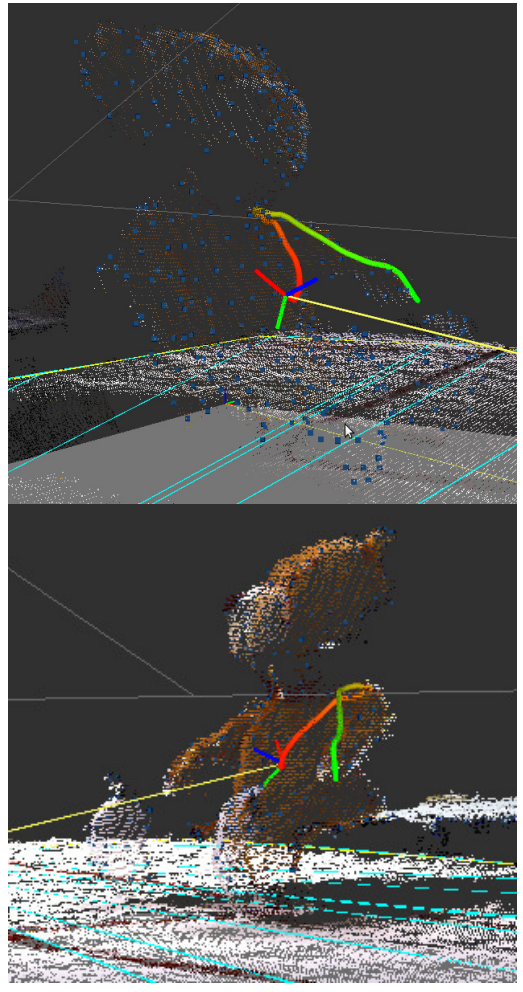

(c) The teddy bear has a very irregular shape, but still geodesic flows are found.

Fig. 3: Geodesic flows on object surfaces captured by an RGBD sensor. The color of the flow goes from red, i.e. the initial contact point, to green, i.e. the final position, according to the predefined length of the curve.

therefore, the labeling goes for a positive, null or zero value, e.g. 1, 0 and -1 , for points that are outside, on, or inside the surface, respectively. The set containing the points on the surfaces are directly in the visual data (Subsection II-A). The set containing points outside the surface is generated by estimating the outward normal over the previous set, and then, displacing the points along the normal. The set containing points inside could be created using a similar method, however, only one inside the surface proved to be enough. Thus, let the vector $\mathbf{y}=\left(y_{1}, \ldots, y_{i}, \ldots, y_{s}\right)^{\top}$ contains the labels corresponding to the joined point set, and covariance matrix $K_{\mathbb{R}_{3}}$ with elements $k_{i j, \mathbb{R}_{3}}=k_{\mathbb{R}_{3}}\left(\mathbf{d}_{i j}, \sigma\right)$, with $\mathbf{d}_{i j}=\mathbf{x}_{i}-\mathbf{x}_{j}$ for all points, the weights are approximated under simplifying assumptions as

$$
\left(\alpha_{1}, \ldots, \alpha_{i}, \ldots, \alpha_{N}\right)^{\top}=K_{\mathbb{R}_{3}}^{-1} \mathbf{y} .
$$

In the case of the friction coefficient function, we would like to model

$$
m(\mathbf{x}) \begin{cases}=\mu, & \text { if } \mathbf{x} \text { is on the surface } \\ <0, & \text { otherwise, }\end{cases}
$$

therefore, the labels are the estimated friction coefficient and negative, e.g. -1 , for points from the tactile and visual data, respectively. Note that, a non-sense value for the friction coefficient is assigned for points that are not explored yet but are known to be on the surface. Like so, the weights are approximated again by arranging a proper label vector $\mathbf{n}=\left(n_{1}, \ldots, n_{i}, \ldots, n_{N}\right)^{\top}$ and covariance matrix $K_{\mu}$ with elements $k_{i j, \mu}=k_{\mu}\left(\mathbf{d}_{i j}, \Sigma\right)$ as

$$
\left(\beta_{1}, \ldots, \beta_{i}, \ldots, \beta_{N}\right)^{\top}=K_{\mu}^{-1} \mathbf{n} .
$$

The contact points during the exploration could potentially be used to refine the object shape representation, but this is useful in the case that they provide radically new information, for instance, when exploring occluded parts of the object, which is clearly a point for future investigation.

\section{B. Exploration paths: Geodesic flows on implicit surfaces}

Paths for exploration should have a good ratio between information gain and exploration length (time or distance). A way to achieve this is to walk in straight lines over the surface to have minimal walking distance. Geodesics can be computed using the implicit function representing the surface and integrating from a point $(x, y, z)^{(0)}$ and a tangent vector $(p, q, r)^{(0)}$ to the surface, until the curve have a predefined length. Thus, given an implicit function $f(x, y, z)$, the geodesic curve equation can formulated in the following six first-order differential equations

$$
\left\{\begin{array}{l}
x^{\prime}=p \\
y^{\prime}=q \\
z^{\prime}=r \\
e^{\prime}=\left(\left(p f_{z}-r f_{x}\right) r+\left(p f_{y}-q f_{x}\right) q\right) L / D \\
f^{\prime}=\left(\left(q f_{z}-r f_{y}\right) r+\left(q f_{x}-p f_{y}\right) p\right) L / D \\
g^{\prime}=\left(\left(r f_{y}-q f_{z}\right) q+\left(r f_{x}-p f_{z}\right) p\right) L / D,
\end{array}\right.
$$


with

$$
\begin{aligned}
L=f_{x x} p^{2}+f_{y y} q^{2}+f_{z z} r^{2}+ & 2\left(f_{x y} p q+f_{y z} q r+f_{x z} p r\right) \\
D=\left(r f_{y}-q f_{z}\right)\left(r f_{y}-q f_{z}\right)+ & \left(p f_{z}-r f_{x}\right)\left(p f_{z}-r f_{x}\right)+ \\
& +\left(q f_{x}-p f_{y}\right)\left(q f_{x}-p f_{y}\right) .
\end{aligned}
$$

The first and second order derivatives of (2) required in (10) are

$$
\frac{\delta f}{\delta \mathbf{x}}=\sum_{i=1}^{N} \alpha_{i} \frac{\delta k_{\mathbb{R}_{3}}(\mathbf{d}, \sigma)}{\delta \mathbf{d}} \frac{\delta \mathbf{d}}{\delta \mathbf{x}},
$$

and

$$
\frac{\delta^{2} f}{\delta \mathbf{x}^{2}}=\sum_{i=1}^{N} \alpha_{i} \frac{\delta^{2} k_{\mathbb{R}_{3}}(\mathbf{d}, \sigma)}{\delta \mathbf{d}^{2}} \frac{\delta^{2} \mathbf{d}}{\delta \mathbf{x}^{2}}
$$

where

$$
\frac{\delta k_{\mathbb{R}_{3}}}{\delta \mathbf{d}}= \begin{cases}-\frac{1}{2 \sigma} \frac{\mathbf{d}^{\top}}{\left(\mathbf{d}^{\top} \mathbf{d}\right)^{\frac{1}{2}}} & \text { if }\left(\mathbf{d}^{\top} \mathbf{d}\right)^{\frac{1}{2}} \leq 2 \sigma \\ \mathbf{0} & \text { if }\left(\mathbf{d}^{\top} \mathbf{d}\right)^{\frac{1}{2}}>2 \sigma,\end{cases}
$$

and

$$
\frac{\delta^{2} k_{\mathbb{R}_{3}}}{\delta \mathbf{d}^{2}}= \begin{cases}-\frac{1}{2 \sigma} \frac{\mathbf{I}_{3} \mathbf{d}^{\top} \mathbf{d}-\mathbf{d d}^{\top}}{\left(\mathbf{d}^{\top} \mathbf{d}\right)^{\frac{3}{2}}} & \text { if }\left(\mathbf{d}^{\top} \mathbf{d}\right)^{\frac{1}{2}} \leq 2 \sigma \\ \mathbf{0}_{3} & \text { if }\left(\mathbf{d}^{\top} \mathbf{d}\right)^{\frac{1}{2}}>2 \sigma\end{cases}
$$

Thus, by integrating (10) using (2), (11), and (12), we obtain geodesic flows $\gamma_{i}$ over the object surface, as shown in Fig. 3.

\section{Contour following: Stiffness controller}

The tactile data acquisition can only be successful when the contact with the object surface is guaranteed. To this end, force and position control is required during the exploration. Considering that there is no exact description of the object shape, executing a pure position control might be dangerous for the equipment. The presented ITs includes a compliant structure for safety, however, when the KUKA arm uses the stiffness controller, it behaves like a spring with defined stiffness and damping parameters. In such manner, we set the parameters to make the PPCC to seem infinitely stiff. The provided control law for the arm for this mode is

$$
\boldsymbol{\tau}=\mathbf{J}^{\boldsymbol{\top}}\left(K_{c}\left(X_{\mathrm{d}}-X\right)+\mathbf{f}_{\mathrm{d}}\right)+B(\mathbf{d})+\boldsymbol{\tau}_{\mathrm{dyn}},
$$

where $X_{\mathrm{d}} \in S E(3)$ is the desired tool frame, $\mathbf{f}_{\mathrm{d}} \in \mathbb{R}^{6}$ is the desired force at the tool, $K_{c} \in \mathbb{R}^{6}$ is the desired Cartesian stiffness of the tool frame specified along the three axes, and d the desired damping behavior.

Concerning the contour following, the origin of the desired tool frame is taken directly from the integration of the geodesic curve. The orientation, instead, is corrected to keep the $z_{r}$ axis of the end-effector reference frame aligned with the normal at the contact point $\mathbf{z}_{\mathrm{c}}$. This is due to the fact that the arm lacks the capability to render any desired Cartesian stiffness in this mode. Thus, since the tip of the ITs is spherical, the orientation correction is equivalent to have a fixed contact point w.r.t. to the sensor frame in a tactileservoing fashion. The error rotation matrix can be readily obtained from angle-axis parameters, with angle

$$
\theta=\cos ^{-1}\left(\mathbf{z}_{c} \cdot \mathbf{z}_{\mathrm{r}}\right)
$$

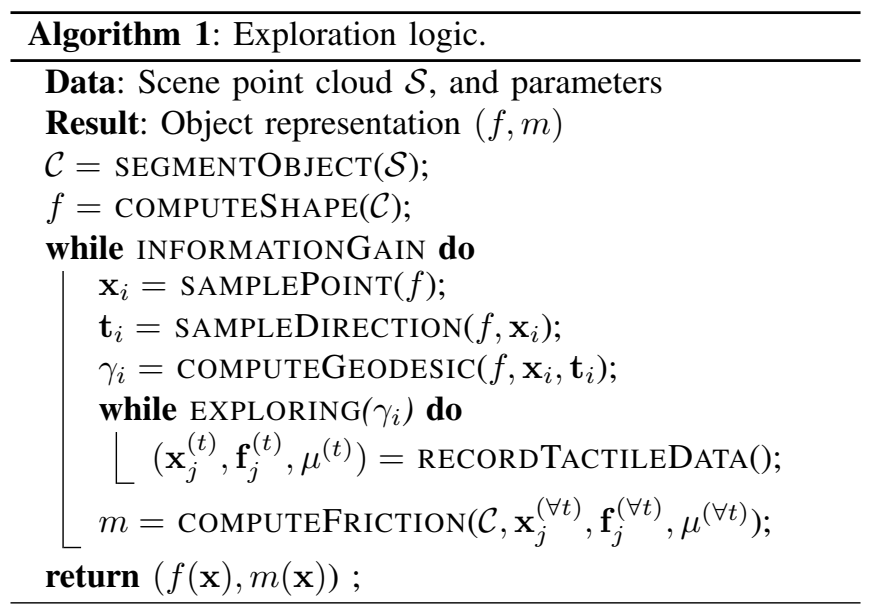

and axis

$$
\mathbf{a}=\frac{\mathbf{z}_{c} \times \mathbf{z}_{\mathrm{r}}}{\sin (\theta)} .
$$

With this, and additionally setting a lower stiff value on the $\mathbf{z}_{\mathrm{r}}$ axis of the tool, as well as a non-zero desired contact force along it, a safe contact and shape accommodation is furnished during the haptic exploration.

\section{Summary: Exploration logic}

The three elements from the previous subsections are looped following the pseudo-code outlined in Algorithm 1, where the arguments of the implicit functions $f, m$ are omitted for clarity. The SEGMENTOBJECT and RECORDTACTILEDATA functions are related to the data acquisition process described in Section II. Functions COMPUTESHAPE and COMPUTEFRICTION are essentially the weight computations in (7) and (9). Function COMPUTEGEODESIC is the integration of (10). The EXPLORING $\left(\gamma_{i}\right)$ loop is performed using the contour following procedure from Section III-C. A clear point for future discussion is how to systematically break the INFORMATIONGAIN loop.

\section{EXPERIMENTS}

\section{A. Implementation details}

The object point cloud segmentation is done in four steps. First, we apply a pass-through filter over the complete point cloud to keep the table and object. Then, a dominant plane is estimated using RANSAC techniques on the filtered cloud. The table is removed, so only the object point cloud remains, which is downsampled to speed up computations. This process is aided by the PointCloud library [17] and the ROS system [18]. The numerical integration of the geodesic flows is performed using the boost $\mathrm{C}++$ odeint library. The F/T sensor used in the ITs is the ATI Nano17 SI-25-0.25. A low-pass filter was applied to the F/T measurements to have a smoother contact estimation. The tip and the PPCC were designed in our lab. The arm where the ITs is mounted on is the 7 DoF KUKA LWR controlled through the Standford Fast Research Interface Library (FRIL). The data acquisition and synchronization interface between the KUKA impedance 

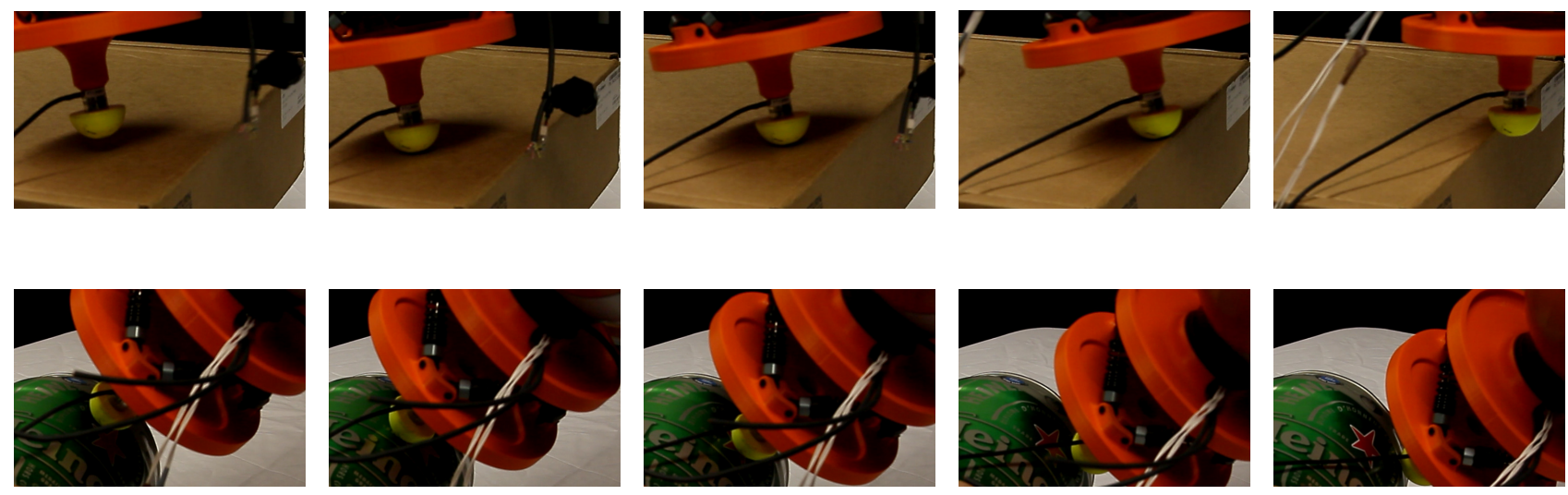

Fig. 4: Haptic exploration over objects. Top: a paperboard box. Bottom: a can.

TABLE I: Parameters used for the experiments.

\begin{tabular}{lc}
\hline Parameter & Value \\
\hline \multicolumn{1}{c}{$R G B D$ sensor, object } & modeling, and \\
Leaf size (downsampling) & $0.02 \mathrm{~m}$ \\
Min cluster size & 500 \\
Surface smoothing factor $\sigma$ & $100 \mathrm{~m}$ \\
Friction smoothing factor $\Sigma$ & 5 \\
Int. fixed-step Runge-Kutta & 0.0001 \\
Geodesic flow length & $15 \mathrm{~cm}$ \\
\hline \multicolumn{1}{c}{$K U K A$ lwr, ITs, and contour following } \\
Robot cycle time & $2 \mathrm{~ms}$ \\
Cartesian stiffness $(\{\mathcal{E}\})$ & $(10,10,2,5,5,5) \cdot 10^{2}[\mathrm{~N} / \mathrm{m}, \mathrm{Nm} / \mathrm{rad}]$ \\
Desired force in $z_{\mathcal{E}}$ & $5 \mathrm{~N}$ \\
Orientation correction gain & 0.001 \\
ITs force threshold & $0.1 \mathrm{~N}$ \\
Tip radius & $0.02 \mathrm{~m}$ \\
F/T filt. low-pass cutoff freq. & $5 \mathrm{~Hz}$ \\
\hline
\end{tabular}

controller, the RGBD sensor and the 6-axes F/T sensor were developed in $\mathrm{C}++$. The relevant parameters used for the experiments are shown in Table I.

\section{B. Results and discussion}

In Fig. 4, we show snapshots of the haptic exploration for two objects, a cardboard box and an aluminum can. The maximum distance between the planned and executed trajectories goes up to $2 \mathrm{~cm}$ in the box, and $0.5 \mathrm{~cm}$ in the can, caused, for instance, by the extrinsic camera calibration, object deformation, and F/T measurements errors. The force measured along $\mathbf{z}_{\mathrm{c}}$ is used to determine the contact state of the trajectory to properly estimate the friction coefficient. Both are plotted in Fig. 5 for a single trajectory. We get a mean value of $\mu=0.62$ and $\mu=0.36$ for the box (ABS/Paperboard) and the can (ABS/Metal), respectively. In Fig. 6, the object shape with the friction coefficient estimation is shown after the haptic exploration along the geodesic flow. The points on the surface are obtained by sampling (2) and the color is obtained by evaluating (4), confirming that the methodology is capable of representing both visual and tactile data.
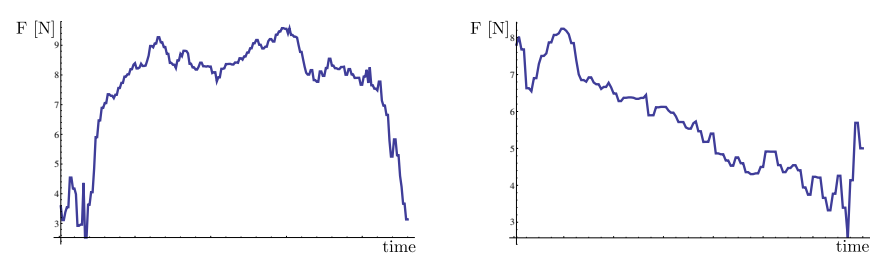

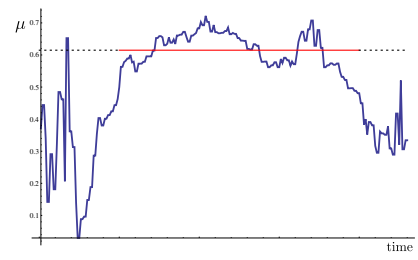

(a) Exploration over the box. Material: Paperboard.

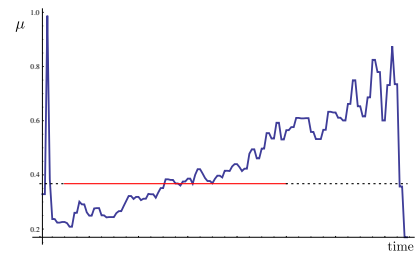

(b) Exploration over the can. Material: Aluminum.
Fig. 5: Relevant data recorded during the haptic exploration. Top: Force along $\mathbf{z}_{\mathrm{c}}$ used to detect the contact and noncontact states for. Bottom: Friction coefficient $\mu$, the red line marks the mean value during the contact state.

\section{CONCLUSIONS}

This work proposed a novel object representation and the exploration strategy to instantiate it. The added value with respect to previous approaches is the consideration of the friction coefficient within the representation. This added information is essential to relevant robotic fields such as grasp planning and manipulation using mechanical hands, and potentially useful in object recognition, in a similar way as [19] recognizes textures. Whereas the visual data was acquired using a well-known RGBD sensor, the tactile data demanded a crude but effective Intrinsic Tactile sensor mounted on a robot's end-effector, acting as an haptic exploratory probe. The exploration is planned in minimal length paths over the object surface, i.e. geodesic flows, to looking to increase the information gain and exploration length ratio. The actual contour following used a stiffness controller for safety, and shape accommodation while ensuring a continuous contact during the exploration. The preliminary experimental results on a single-finger-like fashion revealed a valid approach to 


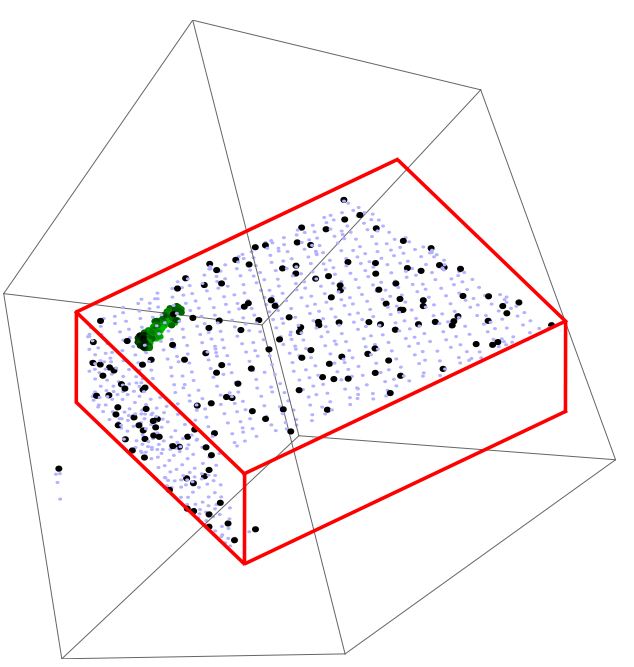

(a) Box

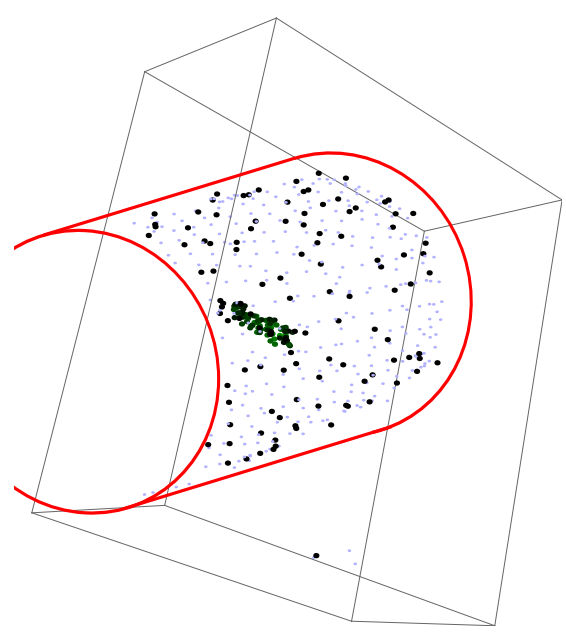

(b) Can

Fig. 6: Points on the object surface are colored with the estimated value of the friction coefficient, that is, the greener the higher, the darker the lower. Note that near the explored trajectory, the friction coefficient is non-zero even when the points are not exactly on the trajectory. For clarity, the actual surface points are smaller and shown in light blue, and an approximated wireframe of the object in red.

have both visual and tactile data in a single probabilistic framework using Gaussian processes.

Several points deserve further attention, such as how to generate points outside/inside the surface for a generic case, or the decision on when to end the exploration if no more information is gained, or how a different contour following controller can affect the quality of acquired data. Future work involves exploring the unseen part of the object, as well as the use of a dexterous hand equipped with ITs at the fingertips, to investigate the limitations due to a reduced mobility, and possibly, the benefits of a parallel tactile data acquisition system.

\section{ACKNOWLEDGEMENT}

This work is supported by the grant no. 600918 "PACMAN" - Probabilistic and Compositional Representations of Object for Robotic Manipulation - within the FP7-ICT-20119 program "Cognitive Systems". European Research Council and under the ERC Advanced Grant no. 291166 SoftHands (A Theory of Soft Synergies for a New Generation of Artificial Hands).

Authors would like to thank Manuel Bonilla for his support in the code implementation and the valuable comments of the reviewers.

\section{REFERENCES}

[1] M. Cutkosky, R. Howe, and W. Provancher, "Force and tactile sensors," in Springer Handbook of Robotics, B. Siciliano and O. Khatib, Eds. Springer Berlin Heidelberg, 2008, pp. 455-476.

[2] W. E. L. Grimson and T. Lozano-Perez, "Model-Based recognition and localization from sparse range or tactile data," The Int. J. of Rob. Res., vol. 3, no. 3, pp. 3-35, Sept. 1984.

[3] P. Allen and R. Bajcsy, "Robotic object recognition using vision and touch," in Proc. of the 9th Int. Joint Conf. on Artif. Intel., 1987, pp. 1131-1137.
[4] J. Ilonen, J. Bohg, and V. Kyrki, "Fusing visual and tactile sensing for 3-D object reconstruction while grasping," in Proc. of the IEEE Int. Conf. on Rob. and Aut., 2013, pp. 3547-3554.

[5] M. Chalon, J. Reinecke, and M. Pfanne, "Online in-hand object localization," in Proc. of the IEEE/RSJ Int. Conf. on Intel. Rob. and Sys., 2013, pp. 2977-2984.

[6] R. Bajcsy, "What can we learn from one finger experiments?" in International Symposium on Robotics Research, 1984.

[7] A. Bicchi, J. K. Salisbury, and D. L. Brock, "Contact sensing from force measurements," The Int. J. of Rob. Res., vol. 12, no. 3, pp. 249-262, June 1993

[8] X. Song, H. Liu, J. Bimbo, K. Althoefer, and L. D. Seneviratne, "Object surface classificaiton based on friction properties for intelligent robotic hands," in World Automation Congress (WAC), 2012, 2012, pp. $1-5$.

[9] K. Hang, F. T. Pokorny, and D. Kragic, "Friction coefficients and grasp synthesis," in Proc. of the IEEE/RSJ Int. Conf. on Intel. Rob. and Sys. IEEE, Nov. 2013, pp. 3520-3526.

[10] R. Detry and J. Piater, "Continuous Surface-Point distributions for 3D object pose estimation and recognition," in Computer Vision ACCV 2010, ser. Lecture Notes in Computer Science, R. Kimmel, R. Klette, and A. Sugimoto, Eds., 2011, vol. 6494, ch. 45, pp. 572-585.

[11] S. Dragiev, M. Toussaint, and M. Gienger, "Gaussian process implicit surfaces for shape estimation and grasping," in Proc. of the IEEE Int. Conf. on Rob. and Aut., May 2011, pp. 2845-2850.

[12] S. El-Khoury, M. Li, and A. Billard, "On the generation of a variety of grasps," Rob. and Aut. Sys., vol. 61, no. 12, pp. 1335-1349, 2013.

[13] CRC Handbook of Chemistry and Physics, 94th Edition, 94th ed. CRC Press, June 2013.

[14] H. Yoshizawa, Y. L. Chen, and J. Israelachvili, "Fundamental mechanisms of interfacial friction. 1. relation between adhesion and friction," J. Phys. Chem., vol. 97, no. 16, pp. 4128-4140, Apr. 1993.

[15] C. E. Rasmussen and C. K. I. Williams, Gaussian Processes for Machine Learning. The MIT Press, 2006.

[16] "NUKLEI: Kernel methods for SE(3) data (a c++ machine-learning library)," http://nuklei.sourceforge.net/.

[17] R. B. Rusu and S. Cousins, "3D is here: Point cloud library (PCL)," in Proc. of the IEEE Int. Conf. on Rob. and Aut., 2011, pp. 1-4.

[18] "ROS: Robot operating system," www.ros.org.

[19] P. Dallaire, P. Giguere, D. Émond, and B. Chaib-draa, "Autonomous tactile perception: A combined improved sensing and bayesian nonparametric approach," Rob. and Aut. Sys., 2014. 